
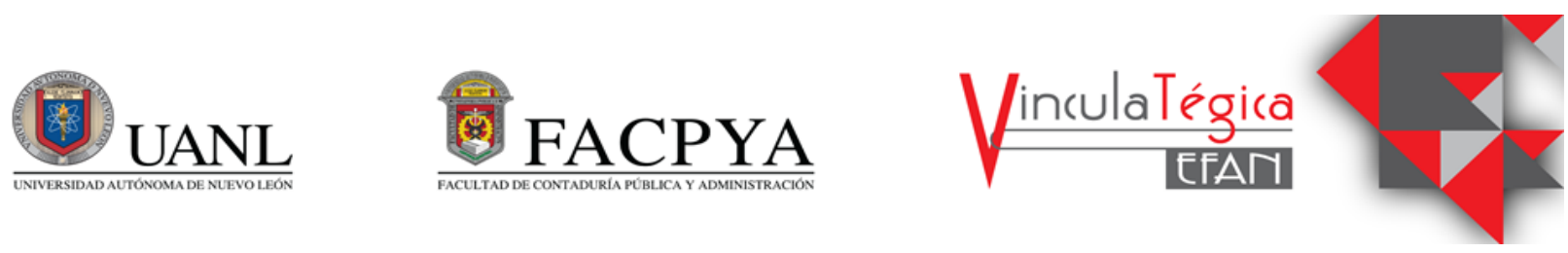

\title{
Decisiones de inversión, desempeño económico, colaboración y apertura de información en tiempos de Covid-19
}

\author{
Pablo Arrona Palacios ${ }^{1}$ \\ ${ }^{1}$ Universidad Autónoma de Nuevo León, Facultad de Contaduría Pública y Administración Monterrey, Nuevo León, \\ México,parronapo@uanl.edu.mx, Av. Universidad S/N Col. Ciudad Universitaria, (+52) 8183204080.
}

Información del artículo revisado por pares

Fecha de aceptación: junio-2021

Fecha de publicación en línea: diciembre-2021

DOI: https://doi.org/10.29105/vtga7.1-137

\begin{abstract}
Resumen
Desde la publicación de la teoría de la selección del portafolio de Markowitz 1952 se han creados diversas estrategias de inversión; sin embargo, no todas de ellas ofrecen un rendimiento excesivo por periodos largos de tiempo; esto puede deberse a variaciones en la economía y a factores externos. El articulo pretende cumplir dos objetivos: el primero es realizar una revisión a la literatura sobre decisiones de inversión durante Covid-19, evaluando factores internos y externos sobre los efectos económicos relacionados en algunas empresas mexicanas ganadoras. Con lo anterior se aportarán estrategias de inversión con miras a entender para así mejorar el desempeño financiero de los individuos inversionistas sobre tiempos de incertidumbre en el terreno internación y nacional. El segundo objetivo es: una examinación a la filología sobre compartir información en ambientes de volatilidad que podrían mejorar la colaboración y apertura de información entre los individuos y empresas.
\end{abstract}

Palabras clave: Covid-19, Decisiones de inversión, desempeño económico, colaboración y apertura de información.

\section{INTRODUCCIÓN}

La Copa del Mundo de 1966 marcó un año de bajo desempeño para fútbol brasileño. El ganador de los dos torneos anteriores, fue eliminado en la primera vuelta, y su jugador estrella, Pelé, falló en su actuar; cometiendo faltas frecuentes y flagrantes, amenazó con no volver nunca a la Copa del Mundo. Muchos se preguntaron si los días de gloria de Brasil habían terminado. Sin embargo, cuatro años más tarde, Brasil volvió a ganar, con tanta gracia y estilo que el equipo de 1970 no solo es ampliamente considerado como el mejor equipo que jamás haya salido al campo, y para coronar el hito Pelé fue nombrado jugador del torneo.

e-ISSN: $2448-5101$ este cambio requirió innovaclinculaTégica EFftedstro
Since the publication of Markowitz's 1952 portfolio selection theory, various investment strategies have been created; however, not all of them offer excessive performance for long periods of time; this may be due to variations in the economy and external factors. This article pretends to accomplish two objectives: the first one is to do a review on published journals about investment decisions under Covid-19 evaluating external factors on the related economic effects on some Mexican winning companies. By fulfilling it, we will contribute with investment strategies in order to understand and to improve the financial performance of individual investors over times of uncertainty in both international and national. The second objective is a literature review on sharing information in volatility environments that could improve collaboration and open information between individuals and companies.

Keywords: Covid-19, Investment decisions, economic performance, collaboration and information openness JEL: M21, D81.

requería construir un equipo con alto nivel de cohesión, incluso contemplando que la mayor parte de la lista de jugadores tuvo rotación. Requirió liderazgo, tanto en la gestión como en el campo. El resultado: reinventando todo, llegó Brasil más fuerte.

A medida que las empresas de todo el mundo desarrollen estrategias al respecto de cómo pueden regresar de un escenario tormentoso infligido por el coronavirus, el camino de Brasil del fracaso al triunfo proporciona elementos a considerar para el pensamiento crítico de dicha estrategia.

Covid-19 ha probado rigurosamente

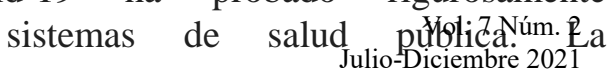


recuperación de Covid-19 pronto probará nuestros sistemas económicos. La innovación será tienen un papel importante que desempeñar en la recuperación de las secuelas del coronavirus.

Los efectos adversos inmediatos que tiene la crisis del COVID-19 para las pequeñas y medianas empresas existentes (PYMES) en términos de su capacidad para mantener los niveles de personal, evitar problemas de flujo de caja y prevenir bancarrotas generalizadas a raíz del gran bloqueo (OCDE, 2020). El trabajo empírico de todo el mundo muestra que tantos como la mitad de todas las pequeñas empresas han dejado de operar temporalmente desde el cierre y hasta el $60 \%$ de las pymes corren el riesgo de quedarse sin reservas de efectivo (Bartik et al., 2020),

La financiación, así como las decisiones de inversión son fundamentales para las empresas emergentes (Cassar, 2004). Sin embargo, debido a la falta de transparencia informativa de la naturaleza de las empresas emergentes innovadoras orientadas al crecimiento lecciones aprendidas valiosas pueden ser compartidas con otras PYMES de tal forma que puedan subsistir.

Por ello el objetivo del artículo es aportar en dos vertientes con respecto al panorama económico: en primera instancia a través de literatura sobre estrategias de inversión en tiempos de crisis en tiempos de COVID-19 y en su segunda parte ideas, conceptos y definiciones del cómo gestionar la apertura, colaboración e innovación que ayude a la recuperación que todo el mundo espera durante y después del COVID19.

\section{MARCO TEÓRICO}

Los últimos 20 años han estado marcados en gran medida en tres crisis globales en los mercados de capitales: la burbuja de las punto.com en 2001, la crisis financiera de $2008 \mathrm{y}$, en la actualidad, la crisis del COVID-19.

Durante la burbuja de la empresa punto.com de la década de 1990, muchos participantes del mercado cuestionaron el valor de información financiera básica para la toma de decisiones de inversión. Se negociaron acciones a un múltiplo récord de ganancias. Esto llevo a que muchas empresas que no obtenían beneficios vieron cómo el precio de sus acciones aumentaba de manera muy rápida en la segunda mitad de la década de 1990. Un número de estudios académicos han documentado una disminución en la relación lineal entre ganancias de las empresas y el rendimiento de las acciones (Brown, \& Lo, \& Lys, T. 1999, Ely, \& Waymire, 1999, Francis, J.,
\& Schipper, K. 1999) Algunos argumentaron que las ganancias ya no importaban y que otras métricas, como el número de clics o visitas a la página era más apropiado en la nueva economía (Penman 2002). Diversos académicos argumentaron que normas contables deficientes contribuyeron al mercado alcista de la década de 1990 (Krugman, 2004)

El período de la burbuja de la década de 1990 `s como una cadena en forma similar a una pirámide en la que las inversiones de impulso desplazaron a las inversiones fundamentales (Penman 2002)

La gran crisis financiera de 2008 ahora se considera una de las más largas y más crisis económicas importantes que el mundo haya visto jamás (Bordo \& Haubrich, 2017) Ha cambiado drásticamente el entorno empresarial del siglo 21 que ya había visto las turbulentas olas de la revolución digital, en el contexto de una globalización cada vez mayor.

Muchos factores contribuyeron a esta crisis financiera, en particular un aumento de la deuda debido a la introducción de nuevos instrumentos financieros, la creación de una burbuja inmobiliaria (burbuja hipotecaria) irresponsable, toma de riesgos y supervisión negligente (Hausman, \& Johnston, 2014). Los principales efectos de la crisis han sido una disminución (o en algunos casos crecimiento más lento) en los países desarrollados, desempleo persistentemente alto, des apalancamiento continuo en el sector privado, grandes déficits y deuda del sector público, una mayor influencia de la política en la economía, una marcada disminución de la inflación, baja tasa de interés, y un acelerado alejamiento de las dinámicas de crecimiento y prosperidad (El-Erian, 2010)

Tan, \& Mathews, (2010) Comentan que el resultado de la crisis financiera del 2008 se puede interpretar como destrucción creativa que es una forma característica del desarrollo capitalista con una serie de altibajos que crean oportunidades de reestructura.

La última y actual crisis fue provocada por la pandemia COVID-19 y sus consecuencias son apenas previsibles. En el corto plazo, se observó un descenso en los mercados de capitales en el primer trimestre del año 2020, pero los precios se recuperaron en el tercer trimestre de 2020. Los estudios al respecto son todavía escasos debido a la actualidad de la crisis.

En México, hay trabajos como el de Salas et al. (2020) el cual estudia la distribución del ingreso laboral y la pobreza a partir de datos de la ENOE. Establecen que la pandemia afectó tanto a aquellos 
cuyos trabajos son informales y no tienen protección social, como la vida de los trabajadores formales. En su estudio que la pobreza podría aumentar hasta $18 \%$ debido a la caída de los ingresos en los grupos más vulnerables del país.

Por ello es importante documentar de visiones estratégicas de inversión y de apertura de conocimiento que ayuden a la sociedad en lo general a modificar patrones en su beneficio.

\section{Metodología}

\subsection{Para ambos objetivos: análisis teórico del estudio en el tema.}

El tipo de metodología usar en el artículo es descriptiva, debido a que se hará una revisión de los diferentes estudios o artículos que se han realizado al respecto sobre decisiones de inversión durante COVID 19 y, colaboración y apertura de información durante COVID 19 a través del buscador de google académico (google scholar). Para esto se pretende responder las siguientes preguntas: ¿cuáles son los principales países donde se ha escrito del objetivo?, ¿quiénes son los principales autores? y en qué año se publicó.

Se encontró poca documentación en el motor de búsqueda al respecto de los objetivos planteados en este artículo se presentan a continuación los resultados obtenidos de búsquedas con comillas para encontrar similitud exacta; realizando el inglés y español.

Con respecto al primer objetivo decisiones de inversión durante COVID 19 solamente se encontró literatura en Ingles. Por ello y ante la escasa literatura sobre el tema se revisará sobre estrategias ganadoras en tiempos de crisis, las cuales ayudaran a generar líneas de investigación sobre el tema a medida que la pandemia avance tanto en México como en otros países.

Tabla 1 Búsqueda del termino en google académico.

\begin{tabular}{|c|c|c|}
\hline Autor & País/ Año & $\begin{array}{c}\text { Investment decisions } \\
\text { during COVID 19 }\end{array}$ \\
\hline $\begin{array}{c}\text { Pena-Marin, } \\
\text { J., Adaval, R., } \\
\text { \& Shen, L. }\end{array}$ & $\begin{array}{c}\text { Estados } \\
\text { Unidos/ } \\
2020\end{array}$ & $\begin{array}{c}\text { Fear in the Stock } \\
\text { Market. How } \\
\text { COVID-19 Affects... }\end{array}$ \\
\hline Badawy, H. & $\begin{array}{c}\text { Egipto/ } \\
2021\end{array}$ & $\begin{array}{c}\text { Financial Reporting } \\
\text { Quality during } \\
\text { COVID-19... }\end{array}$ \\
\hline Hirvonen, T. & $\begin{array}{c}\text { Finlandia/ } \\
2021\end{array}$ & $\begin{array}{c}\text { Behavioural Finance } \\
\text { in Financial Crises.. }\end{array}$ \\
\hline
\end{tabular}

Con respecto al segundo objetivo colaboración y apertura de información durante COVID 19 solamente no se encontraron artículos con dicha exactitud. Por ello y ante la escasa literatura sobre el tema se revisará sobre estrategias recuperación desde la innovación y colaboración, dicho tema ayudará a generar líneas de investigación en un futuro a medida que la pandemia avance tanto en México como en otros países.

\section{COVID 19 \& Estrategias de inversión}

El 11 de marzo de 2020, la Organización Mundial de la Salud (OMS) manifestó de forma oficial que el brote de coronavirus (COVID-19) era una pandemia mundial1. Al 27 de marzo de 2020, el número de casos confirmados superó los 500.000 y sigue aumentando (OMS, 2020). Más de 170 países se ven afectados, y EE. UU. Tiene la mayoría de los casos confirmados. El brote ha tenido impactos económicos importantes y significativos a corto plazo en casi toda la economía de planeta, dado que en una gran cantidad de países adoptan políticas estrictas o semi-estrictas de cuarentena, esto ocasionar que sus actividades económicas son limitadas y diferenciadas en cada país. Entre algunas de las muchas consecuencias de largo plazo de esta pandemia se encuentra el desempleo masivo y la quiebra de negocios de todos los tamaños. Algunas industrias en lo particular, como el turismo y la aviación, enfrentarán dificultades tanto económicas como físicas derivadas de la pandemia que aqueja al mundo cuya determinación final está aún lejos de ser establecida.

Con respecto al impacto económico y su recuperación, la contribución de Narayan, et al. (2021), quienes analizan el impacto de las medidas gubernamentales sobre el exceso de rentabilidad en los mercados de los países del G7, debe tenerse en cuenta. Según Narayan, et al. (2021), las medidas gubernamentales parecen tener un efecto positivo en la rentabilidad.

El segundo punto sobre los efectos físicos, Hetkamp et al. (2020) nos comentan al respecto del análisis de los efectos psicológicos, como los trastornos del sueño, la ansiedad y la bolsa de valores alemana (DAX). El objetivo de esta encuesta basada en la web era evaluar la carga de salud mental del público alemán durante un período de 50 días después del brote de COVID19. 16,245 individuos respondieron con respecto a los trastornos del sueño, el miedo a COVID-19 y el trastorno de ansiedad generalizada. Los datos se pusieron en relación con las tasas de infección, el número de muertes y el índice bursátil. Sin embargo, no se pudieron derivar relaciones significativas.

Que puede hacer el inversionista promedio 
ante esta situación; numerosos estudios científicos muestran que la gestión activa de los portafolios de inversión puede superar estrategias pasivas. (Rachev, et al 2007). De tal suerte que el desempeño que se puede gestionar en tiempos de crisis mediante las estrategias basadas en datos fundamentales. En este contexto, se seleccionaron contribuciones de la literatura del área de estrategias de valor, impulso y al respecto de las burbujas de precios de activos.

\subsection{Estrategias de valor}

El contenido de las estrategias de valor es la investigación de las relaciones económicas que son responsables de la fijación de precios justos en los mercados de valores. Este precio justo podría verse influenciado por micro factores o relaciones macroeconómicas, tales como desarrollo económico, consumo privado, tasas de inflación o tasas de interés (Langenstein, \& Warias 2021) Debido al hecho de que los mercados son inherentemente ineficientes (Malkiel, \& Fama, E. F. 1970) una estrategia de inversión fundamental puede generar un rendimiento superior.

Los primeros estudios que se realizaron y muestran que las acciones con una alta relación beneficio-precio o altos valores de las acciones contables con relación a su precio de mercado generan rendimientos más altos (Basu,1997). La explicación de este fenómeno lo abarcan Fama \& French (1992) al comentar que estos altos rendimientos superiores, quienes argumentan que es debido al mayor riesgo en las estrategias de valor, los rendimientos más altos se traducen en una alta prima de riesgo (French 1992). Lakonishok, Shleifer y Vishny (1994) explican estos rendimientos excesivos por la capacidad de los inversionistas para identificar activos infravalorados.

\subsection{Estrategias de momento}

El enfoque de la estrategia de momento como estrategia de inversión pro cíclica radica en la hipótesis de que las acciones ganadoras del pasado probablemente se desarrollarán en la misma dirección en el futuro cercano. Lo mismo se supone para el desempeño de las acciones perdedoras (Bajgrowicz \& Scaillet 2012, Asness, \& Moskowitz, \& Pedersen 2013). Las reglas comerciales técnicas de fuerza relativa de acuerdo con Levy (1967) proporcionan la base para este supuesto. La estrategia de momento puede ofrecer a los inversionistas la oportunidad de superar el mercado (Rachev, et al 2007). Los primeros estudios empíricos sobre la estrategia de momento se refieren al mercado estadounidense.

Entre 1960 y 1965 (Levy 1967) examinó una muestra de 200 acciones de la Bolsa de Nueva York (NYSE) cuyos precios de cierre semanales durante un período de 260 semanas se utilizaron como base de datos para el análisis y proporcionó evidencia científica del éxito de la estrategia de momento (Jegadeesh \& Titman, 1993) Demostraron que una estrategia de inversión genera un exceso de rentabilidad de hasta el $12,01 \%$ anual en promedio para el mercado estadounidense. Al mismo tiempo, sin embargo, el estudio deja claro que el exceso de rendimiento de la inversión universo se reducen hasta la mitad en el período de observación a largo plazo; 24 meses después del período de espera.

August \& Schiereck \& Weber (2000) analizaron los datos de precios de 418 acciones semanalmente. y mensualmente, que cotizaron en el mercado Aleman entre 1973 y 1997. Se demostró una estrategia de impulso exitosa para rendimientos ajustados al riesgo. De acuerdo con los estudios existentes, se requiere un período de formación y prueba de seis y doce meses, los cuales en base a la evidencia empírica demuestra ser prometedor. Es de notar que, en comparación con otros estudios, los ganadores y los perdedores muestran un exceso de rentabilidad del 6,12\% y el $6,25 \%$, respectivamente. Además, los patrones para el retorno estacional se vuelven evidentes. Estudios previos realizados en otros mercados de alto volumen de operación como el mercado alemán se refieren igualmente a la superioridad de la estrategia de impulso (Choi et al 2015, Bohl et al 2016)

\subsection{Burbujas de precios en las inversiones}

En sentido amplio de la palabra, la literatura usa el término burbuja de precios de activos para describir una situación en el mercado en el que los valores de mercado se desvían considerablemente de los valores fundamentales (Shiller 2000, Garber, P. M. 2001). Al tener una definición sobre las burbujas, su valoración es importante. La literatura nos aporta sobre ese tema a ese problema; es decir el conocer una determinación exacta del valor fundamental; para dicho enfoque Rosser, J. B. (2000) nos aporta que en el momento de la ocurrencia de la burbuja es casi imposible de determinar. Siegel (2003) sugiere medir una burbuja de precios de activos en función de las desviaciones en los rendimientos esperados y realizados durante un período de tiempo definido. 
Esto solo se puede hacer en retrospectiva, ya que los retornos no se pueden predecir con certeza. Pastor \& Veronesi (2006) amplían esta perspectiva para incluir incertidumbre; y sugieren tener en cuenta la incertidumbre da una perspectiva más realista para riesgo de flujo de caja. Esto fue cierto para las empresas. en el segmento de internet, biotecnología y telecomunicaciones en la burbuja de las puntocom de 2001.

Factores blandos como capital humano, alianzas estratégicas, inversiones en empresas conjuntas e Internet están incrementando en importancia (Sousa \& Pinho 2014) Estos factores se incluyen en las expectativas del mercado en las proyecciones de flujo de caja. Por ejemplo, un estudio de O'Brien \& Tian (2006) mostró que los analistas financieros eran más optimistas sobre las acciones de Internet durante la burbuja de las puntocom. Fue precisamente en esta fase cuando los valores fundamentales se volvieron irrelevantes para los inversionistas.

\subsection{Evidencia reciente}

Langenstein, \& Warias, (2021) realizan un análisis basado en información financiera mostrada por empresas del S\&P500 y del STOXX europeo, tuvo como base a todas las empresas. El retorno de la acción de estas empresas fue la variable dependiente, como variables independientes se usaron la capitalización de mercado, la relación precio a ganancias, precio de mercado en relación al precio de libros, la rentabilidad de los dividendos, el flujo de efectivo en relación a las ventas y el precio en relación a las ventas.

En base a la información anterior se clasificaron a las empresas en cuartiles para su identificación y medición.

Figural: Promedio de retornos por cuarto del SP\&500 Q1 1998 - Q3 2020)
Average Quaterly Performance STOXX EUROPE $600($ Q1 1998 - Q3 2020)

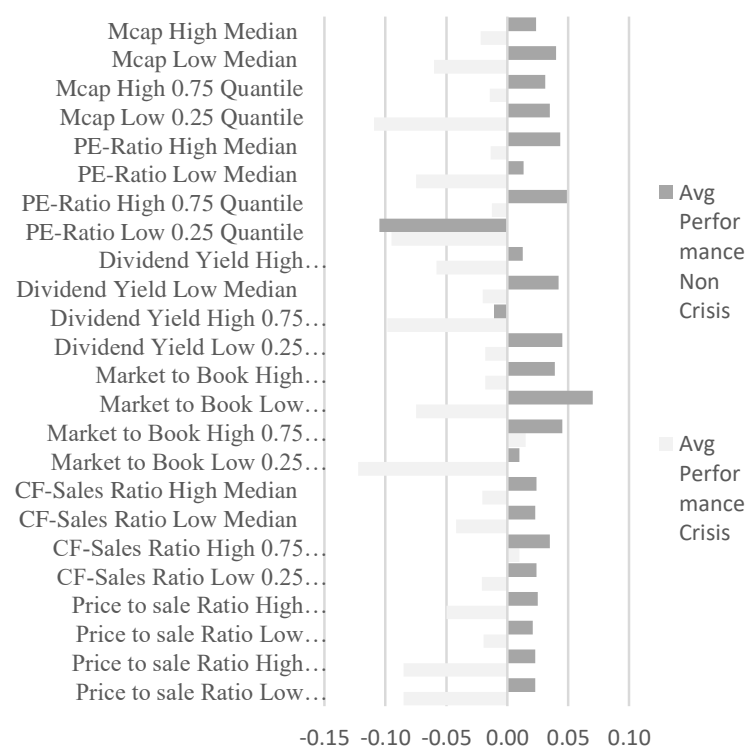

Fuente: Langenstein, \& Warias, (2021)

Los autores realizan una separación importante para analizar tendencias en cuanto a empresas "digitales" clasificadas ya sea de software, servicios de IT o equipo de tecnología de dicha forma por "Thomson Reuters Business Clasification Sector" como nota adicional Amazon fue clasificada como digital. Al realizar dicha clasificación se obtienen resultados interesantes:

Figura2: (Promedio de retornos por cuarto de las crisis $2000 / 2001-2008 / 2009$ - 2020 del SP\&500)

Quaterly Returns in Crisis S\&P 500

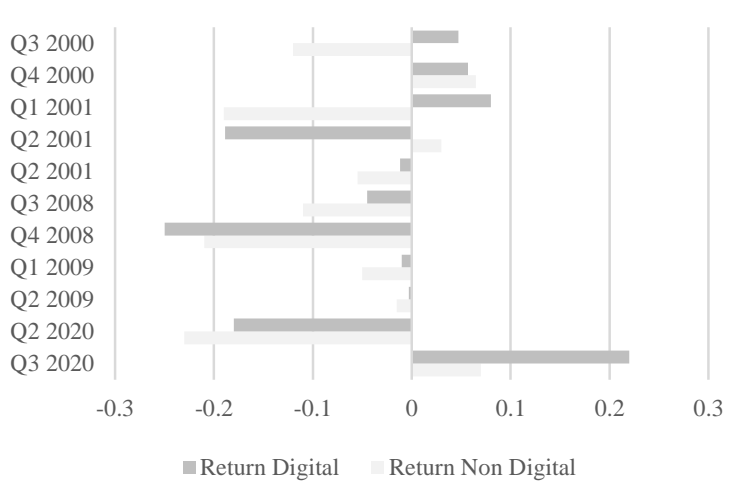

Fuente: Langenstein, \& Warias, (2021)

Los datos enfocados en empresas digitales, muestran que obtuvieron retornos positivos particularmente en el segundo cuarto de la crisis del COVID-19. De manera contrastante las empresas 
digitales tuvieron pérdidas durante la crisis del "dot.com"

\subsection{Casos en México}

Dado que el COVID-19 ha sido declarado pandemia, se espera que su impacto trascienda a no solo un solo país, región o continente. En otras palabras, se espera que afecte la oferta y la demanda mundial de bienes y servicios, entre los que se encuentran los precios de las materias primas y los precios de las acciones. Los estudios han demostrado que la inclusión de activos de materias primas en las carteras de los inversores es capaz de protegerse contra el riesgo, ofreciendo así una mayor rentabilidad, especialmente cuando el mercado está en modo bajista (Öztek and Öcal, 2017; Zhang et al., 2017; and Aït-Youcef, 2018) Para el caso mexicano podemos observar que el mercado tiene aspectos que van orientados a una dependencia del mercado externo en combinación con una protección ante el mercado en declive, observando un comportamiento similar a los estudios mencionados. Dentro de la muestra se seleccionan las empresas ganadoras del 2020 son de clúster minero: Peñoles $(>70 \%)$, Grupo México (>60\%); y de Bebidas como José Cuervo.

El alza en el precio de los metales preciosos a nivel mundial beneficio a las empresas mineras. La empresa Peñoles, tiene en la plata su actividad principal, seguida del oro.

Al buscar una protección para los activos de inversionistas, los metales preciosos son buscados en tiempos de alta incertidumbre, porque son considerados valores de refugio con bajo riesgo. Durante tiempos de dificultades económicas, se observa que los inversionistas exhiben un "efecto de vuelo" hacia activos con alta calidad, con los cuales se reequilibran los portafolios de inversión hacia valores menos riesgosos, como por ejemplo la renta fija (Abel, 1988; Barsky, 1989; Durand et al., 2010). Otra opción estudiada es que los inversionistas han optado por metales preciosos como cobertura o refugio durante condiciones de mercado altamente volátiles. De forma empírica se ha probado que el Oro que posee propiedades de refugio seguro durante la volatilidad extrema en los mercados de valores (Baur \& Lucey, 2010).

Figura3: Dólares americanos por onza troy de ORO índex mundial
Dolares americanos por onza Troy

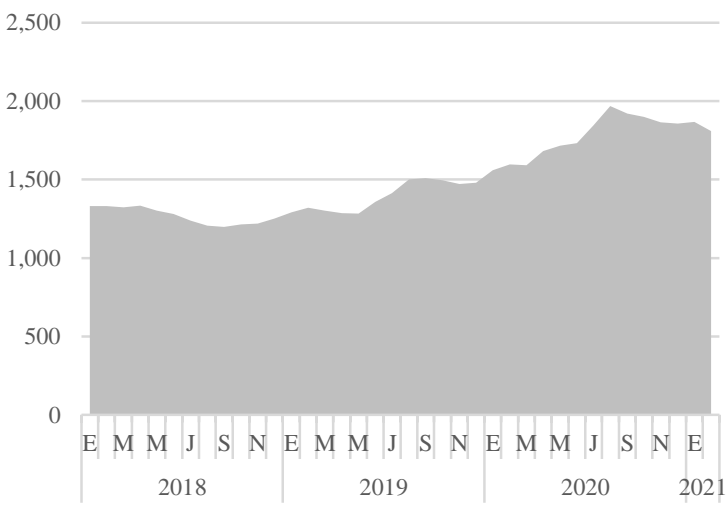

Fuente: Elaboración propia

Otros metales preciosos como la plata, el platino y el paladio exhiben un refugio seguro propiedades durante períodos que el oro no tiene (Lucey \& Li, 2013). Durante el año 2020, el precio del oro y la plata se incrementó en más de un $20 \%$ más del $40 \%$ respectivamente.

Figura4: Dólares americanos por onza troy de PLATA índex mundial.

Dolares americanos por onza Troy de Plata

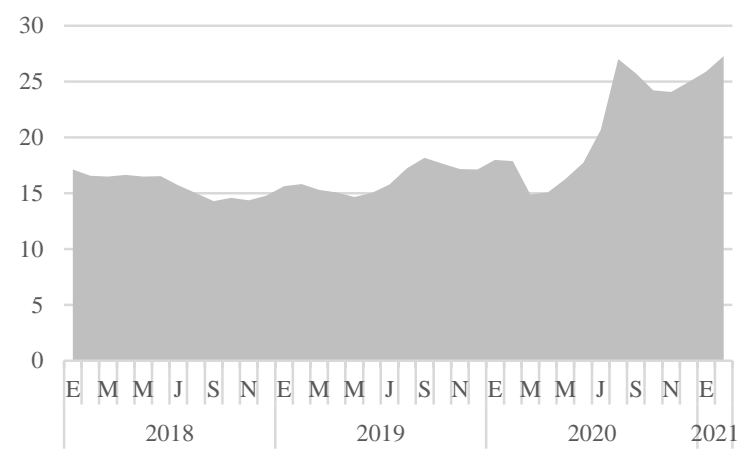

Fuente: Elaboración propia

Grupo México, también minera, es una de las grandes empresas en cuanto a la producción de cobre e refiere. Al ser considerado el cobre un metal del corte de materias primas globales (commodity) según los estudios mencionados por Zhang \& Broadstock, (2018) la interconexión con la crisis global y los mercados de materias primas existe y se refleja como activos seguros para invertir, ocasionando su incremento en el precio. En el año 2021, acumula un avance de más del 27\%

Figura5: Dólares americanos por Tonelada Métrica de Cobre índex mundial. 
Dolares americanos por Tonelada Metrica de Cobre

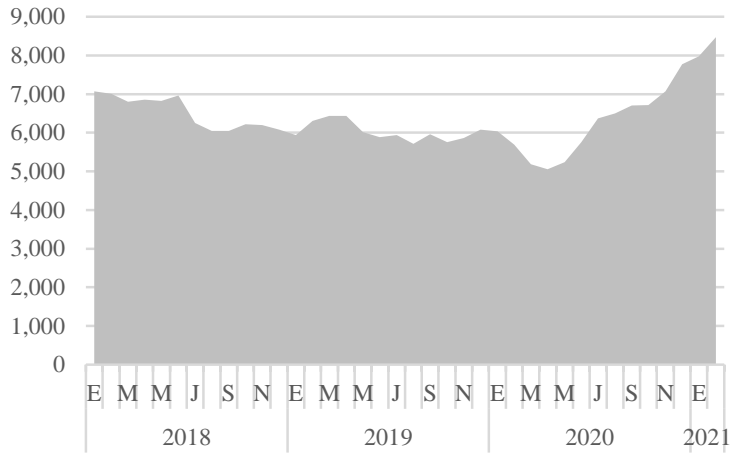

Fuente: Elaboración propia

El encierro durante la pandemia tuvo un efecto en empresas como por ejemplo el Grupo José Cuervo. Weerakoon, Jetelina, \& Knell, (2020) encuestaron a 1,928 participantes autoseleccionados a lo largo de USA los cuales completaron el cuestionario electrónico en los primeros meses de la pandemia. Dicho cuestionario preguntó sobre antecedentes psiquiátricos, conductas en pandemia y los hábitos de bebida. Comentan que los que respondieron habían estado confinados 4 semanas en promedio. $32 \%$ de los que respondieron afirmaban que habían aumentado el consumo de alcohol durante la pandemia y $10 \%$ que habían bajado su consumo. Segregando al respecto de la población del estudio, el $35 \%$ se auto clasificó como "bebedor compulsivo; es decir hombres que consumen más de cinco bebidas alcohólicas en un día o mujeres que beben más de cuatro". El aumento del consumo de alcohol en los bebedores compulsivos fue más alto que en los bebedores no compulsivos; $60 \%$ de los bebedores compulsivos notificaron un aumento adicional del consumo de alcohol, frente a $30 \%$ de los bebedores no compulsivos.

Figura6: Incrementos entre grupo de tiempo de Bebedores (Weerakoon, Jetelina, \& Knell, 2020).
Incrementos entre grupos de Tipo de Bebedores

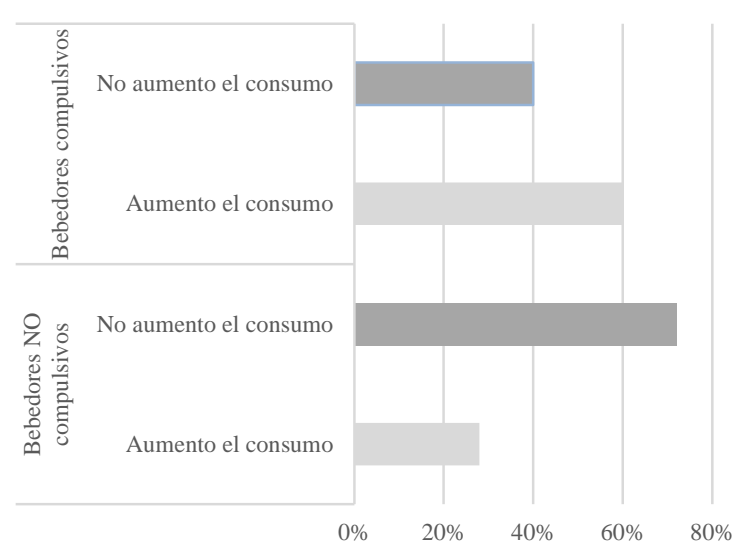

Fuente: Elaboración propia

Para México en su conjunto es importante estar suficientemente informado de las tendencias globales y a los cambios que se están dando en los mercados, de tal forma que se encuentren los espacios convenientes para direccionar las inversiones. A continuación, veremos la forma en que la información ayudara a tomar mejores decisiones.

\section{Apertura en la información}

El repentino suceso del Covid-19 ha provocado una serie de desarrollos alentadores. Un avance ha sido la rápida movilización de científicos, empresas farmacéuticas y funcionarios gubernamentales para lanzar una variedad de iniciativas científicas para encontrar una respuesta eficaz al virus.

Un segundo desarrollo ha sido la liberación de grandes cantidades de información sobre el virus, su propagación y las respuestas humanas a diversas medidas de salud pública. La Fundación Gates, en colaboración con ChanZuckerberg Foundation (CZF 2021) y la Oficina de la casa blanca de Ciencia y Tecnología se han unido para publicar toda la literatura médica sobre el coronavirus.

Lo que estos desarrollos tienen en común es la apertura. En la lucha contra una pandemia, la velocidad es crucial, y cuanto antes sepamos más y podamos para actuar, mejor para todos. Dicha apertura moviliza el conocimiento desde muchos lugares diferentes, lo que hace que nuestro aprendizaje avance y nuestro progreso contra la enfermedad se mueva muy rápido. Tanto fue así que la apertura dio rienda suelta a un ejército de investigadores, trabajando en sus propias instalaciones, a través de diferentes zonas horarias y diferentes países. La apertura también aprovecha 
el capital humano disponible en el mundo para hacer frente a la enfermedad, y también accede al capital físico (como plantas y equipos). Esta apertura corresponde nos lleva a lo que se le puede denominar innovación abierta (Chesbrough, 2003; Chesbrough, 2019).

\subsection{Innovación abierta, ¿Qué es?}

Para comprender mejor cómo funciona la innovación abierta, es útil comparar y contrastar algunos de sus principales mecanismos en tiempos normales y enfrentarlo a nuestra crisis actual. La innovación abierta se define como "una innovación que implica flujos de conocimiento intencionados a través de toda la organización por razones monetarias o no monetarias " (Chesbrough \& Bogers, 2014).

Tiene dos direcciones principales en el flujo de conocimiento: de afuera hacia adentro y de adentro hacia afuera. Estos funcionan un poco de manera diferente en contextos de empresa a empresa (b2b) que en contextos de empresa a consumidor (B2c). Algunos ejemplos rápidos ayudan a aclarar estos mecanismos.

Para los flujos de conocimiento de afuera hacia adentro, Procter \& Gamble lanzó su programa Conectar y desarrollar para solicitar colaboraciones con socios todos en todo el mundo (Huston \& Sakkab, 2006). Otro ejemplo de afuera hacia adentro lo encontramos que NASA lanzó un desafío de innovación abierta al público, y recibió miles de sugerencias de personas para encontrar formas de abordar los peligros en el espacio (Lifshitz-Assaf, 2018).

En el ambiente actual sobre Covid-19, estamos viendo proyectos por ejemplo de crowdsourcing de terapias para manejo de la enfermedad, además de formas de diseñar y fabricar máscaras, desinfectantes de manos, e incluso modificar un respirador para soportar 2 pacientes a la vez.

Por otro lado, para los flujos de conocimiento de adentro hacia afuera en el contexto b2b, Amazon ofreció su infraestructura de TI interna para alojar sitios web y necesidades de TI de clientes externos (Huckman, Pisano \& Kind, 2008). Durante la pandemia de Covid19 Medtronic ha abierto su diseño de ventilador para cualquier persona; publicó sus dibujos de diseño interno para el ventilador y renunció a sus derechos de propiedad intelectual sobre el diseño. En el contexto b2c, Lego permitió a los usuarios y profesores modificar su producto Mindstorms para crear un programa completo de clases de robótica en escuelas intermedias (Afari \& Khine,
2017). Durante la pandemia COVID 19 empresas han convertido sus operaciones de producir solamente alcohol a manufacturar desinfectante de manos

\subsection{Administrar para la recuperación}

Gestionar la innovación es importante, incluso después de que la crisis finalmente pase. La apertura es esencial para responder a la pandemia. A continuación, se muestran algunas prácticas recomendadas, mientras cada empresa traza su curso para recuperarse de la pandemia (Chesbrough 2020). En empresas tecnológicas/ científicas animar a los científicos e ingenieros a involucrarse profundamente con la comunidad científica de forma amplia, permitiendo invertir tiempo y contribuir para la comunidad. Esto podrá traer conocimiento y proporcionar validación adicional para posibles innovaciones en la empresa. Que el experimentar sea el nombre del juego, así como aprender de los experimentos de otros.

Muchos de las soluciones a los problemas más desafiantes pueden encontrarse en diferentes lugares del mundo. Compartir los problemas, junto con su pertinencia científica, técnica y datos, en plataformas abiertas, para que cualquier persona interesada puede ofrecer sus ideas sobre la mejor manera de abordar el problema.

Las buenas ideas pueden provenir de cualquier lugar, esto hacer que la apertura sea un tema que emerja de forma natural en estos tiempos de crisis. La apertura acelerara el proceso de innovación, le permiten aprovechar el conocimiento. Este ciclo creará más experimentos, y por ende genera más conocimiento.

\section{Conclusiones}

Ante la incertidumbre en el panorama actuales es fundamental contar con opciones de inversión basadas en información estudiada con miras a mejorar el desempeño financiero de los individuos inversionistas sobre la toma de decisiones e inversión sobre tiempos de incertidumbre en el terreno internacional y nacional, lo cual es aplicable para individuos como para empresas.

Los resultados observados de la muestra de empresas ganadoras en México durante el 2020 respaldan los hallazgos de varias publicaciones empíricas mencionadas al respecto de que invertir en materias primas, especialmente durante períodos de crisis y turbulentos, sirve como una buena cobertura contra la volatilidad y las caídas 
en los mercados de valores.

Al contar con estrategias probadas empíricamente, viene, en segundo plano la colaboración y apertura de información entre los individuos y empresas; lo cual nos permitirá compartir experiencias, aprendizajes, así como lecciones aprendidas de tal forma que quien reciba el conocimiento le permita tener elementos para mejorar la calidad de sus decisiones. 


\section{REFERENCIAS}

Abel, A. B. (1988). Stock prices under time-varying dividend risk: An exact solution in an infinite-horizon general equilibrium model. Journal of Monetary Economics, 22(3), 375-393. En: https://doi.org/10.1016/0304-3932(88)90004-9

Afari, E., \& Khine, M. S. (2017). Robotics as an educational tool: Impact of lego mindstorms. International Journal of Information and Education Technology, 7(6), 437-442. En: http://www.ijiet.org/show-881038-1.html

Aït-Youcef, C. (2019). How index investment impacts commodities: A story about the financialization of agricultural commodities. Economic Modelling, 80, 23-33. En: https://www.sciencedirect.com/science/article/abs/pii/S0264999317309598

Asness, C. S., Moskowitz, T. J., \& Pedersen, L. H. (2013). Value and momentum everywhere. The Journal of Finance, 68(3), 929-985. En: https://doi.org/10.1111/jofi.12021

August, R., Schiereck, D., \& Weber, M. (2000). Momentumstrategien am deutschen Aktienmarkt: neue empirische Evidenz zur Erklärung des Erfolgs (No. 35294). Darmstadt Technical University, Department of Business Administration, Economics and Law, Institute for Business Studies (BWL). En: https://ideas.repec.org/p/dar/wpaper/35294.html

Badawy, H. (2021). The Effect of Quality and Timeliness of Limited Review Report on Perceived Interim Financial Reporting Quality during COVID-19 Pandemic Crisis: Evidence from Egypt. العلمية المجلة

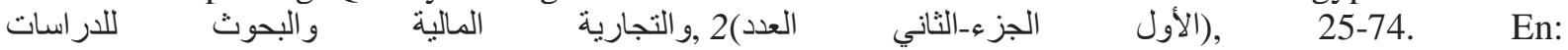
https://journals.ekb.eg/article_146711.html

Bartik, A. W., Bertrand, M., Cullen, Z. B., Glaeser, E. L., Luca, M., \& Stanton, C. T. (2020). How are small businesses adjusting to COVID-19? Early evidence from a survey (No. w26989). National Bureau of Economic Research. En: https://www.nber.org/papers/w26989

Bajgrowicz, P., \& Scaillet, O. (2012). Technical trading revisited: False discoveries, persistence tests, and transaction costs. Journal of Financial Economics, 106(3), 473-491. En: https://doi.org/10.1016/j.jfineco.2012.06.001

Barsky, R. B. (1986). Why don't the prices of stocks and bonds move together? (No. w2047). National Bureau of Economic Research. En: https://www.nber.org/papers/w2047

Basu, S. (1997). The conservatism principle and the asymmetric timeliness of earnings1. Journal of accounting and economics, 24(1), 3-37. En: https://doi.org/10.1016/S0165-4101(97)00014-1

Baur, D. G., \& Lucey, B. M. (2010). Is gold a hedge or a safe haven? An analysis of stocks, bonds and gold. Financial Review, 45(2), 217-229. En: https://doi.org/10.1111/j.1540-6288.2010.00244.x

Bohl, M. T., Czaja, M. G., \& Kaufmann, P. (2016). Momentum profits, market cycles, and rebounds: Evidence from Germany. The Quarterly Review of Economics and Finance, 61, 139-159. En: https://doi.org/10.1016/j.qref.2016.01.003

Bordo, M. D., \& Haubrich, J. G. (2017). Deep recessions, fast recoveries, and financial crises: Evidence from the American record. Economic Inquiry, 55(1), 527-541. En: https://doi.org/10.1111/ecin.12374

Brown, S., Lo, K., \& Lys, T. (1999). Use of R2 in accounting research: measuring changes in value relevance over the last four decades. Journal of Accounting and Economics, 28(2), 83-115. En: https://doi.org/10.1016/S0165-4101(99)00023-3

Cassar, G. (2004). The financing of business start-ups. Journal of business venturing, 19(2), 261-283. En: https://doi.org/10.1016/S0883-9026(03)00029-6

ChanZuckerberg Foundation. CZF (2021). CZI COVID-19 Response. 05/03/2021, de ChanZuckerberg Foundation. En: https://chanzuckerberg.com/covid-19/

Chesbrough, H. W. (2003). Open innovation: The new imperative for creating and profiting from technology. Harvard Business Press.

Chesbrough, H. (2019). Open innovation results: Going beyond the hype and getting down to business. Oxford University Press.

Chesbrough, H., \& Bogers, M. (2014). Explicating open innovation: Clarifying an emerging paradigm for understanding innovation. New Frontiers in Open Innovation. Oxford: Oxford University Press, Forthcoming, 3-28. En: https://ssrn.com/abstract=2427233

Chesbrough, H. (2020). To recover faster from Covid-19, open up: Managerial implications from an open innovation perspective. Industrial Marketing Management, 88, 410-413. En: https://doi.org/10.1016/j.indmarman.2020.04.010

Choi, J., Kim, Y. S., \& Mitov, I. (2015). Reward-risk momentum strategies using classical tempered stable

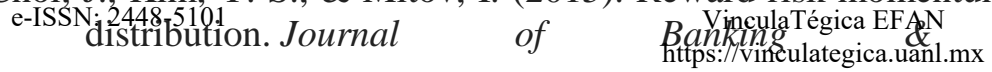

Finance, 58, 194-213Vol. 7 Núm $\mathbb{E}^{2}$ : 
https://doi.org/10.1016/j.jbankfin.2015.05.002

Durand, R. B., Junker, M., \& Szimayer, A. (2010). The flight-to-quality effect: a copula-based analysis. Accounting \& Finance, 50(2), 281-299. En: https://doi.org/10.1111/j.1467629X.2009.00320.x

Ely, K., \& Waymire, G. (1999). Accounting standard-setting organizations and earnings relevance: longitudinal evidence from NYSE common stocks, 1927-93. Journal of accounting Research, 37(2), 293-317. En: https://doi.org/10.2307/2491411

El-Erian, M. A. (2010). Navigating the new normal in industrial countries. Washington, DC: Per Jacobsson Foundation.

Fama, E. F., \& French, K. R. (1992). The cross-section of expected stock returns. the Journal of Finance, 47(2), 427-465.

Francis, J., \& Schipper, K. (1999). Have financial statements lost their relevance? Journal of accounting Research, 37(2), 319-352. En: https://doi.org/10.2307/2491412

Garber, P. M. (2001). Famous first bubbles: The fundamentals of early manias. mit Press.

Hetkamp, M., Schweda, A., Bäuerle, A., Weismüller, B., Kohler, H., Musche, V., ... \& Skoda, E. M. (2020). Sleep disturbances, fear, and generalized anxiety during the COVID-19 shut down phase in Germany: relation to infection rates, deaths, and German stock index DAX. Sleep medicine, 75, 350-353. En: https://doi.org/10.1016/j.sleep.2020.08.033

Hirvonen, T. (2021). Behavioural Finance in Financial Crises: The Case of Covid-19. En: https://aaltodoc.aalto.fi/handle/123456789/107346

Levy, R. A. (1967). Relative strength as a criterion for investment selection. The Journal of finance, 22(4), 595-610. En: https://doi.org/10.2307/2326004

Lifshitz-Assaf, H. (2018). Dismantling knowledge boundaries at NASA: The critical role of professional identity in open innovation. Administrative science quarterly, 63(4), 746-782. En: https://doi.org/10.1177/0001839217747876

Hausman, A., \& Johnston, W. J. (2014). Timeline of a financial crisis: Introduction to the special issue. Journal of Business Research,Volume 67, Issue 1, 2667-2670. En: https://doi.org/10.1016/j.jbusres.2013.03.014.

Tan, H., \& Mathews, J. A. (2010). Identification and analysis of industry cycles. Journal of Business Research, 63(5), 454-462. En: https://doi.org/10.1016/j.jbusres.2009.04.012

Huckman R.S, Pisano C.P and Kind L. (2008). Amazon Web Services. Harvard Business School Case\# 9609-048. En: https://www.hbs.edu/faculty/Pages/item.aspx?num=36511

Huston, L., \& Sakkab, N. (2006). Inside Procter \& Gamble's new model for innovation.

Harvard business review, 84(3), 58-66. En: https://hbr.org/2006/03/connect-and-develop-inside-proctergambles-new-model-for-innovation

Jegadeesh, N., \& Titman, S. (1993). Returns to buying winners and selling losers: Implications for stock market efficiency. The Journal of finance,48(1), 65-91. En: https://doi.org/10.1111/j.15406261.1993.tb04702.x

Krugman, P. (2004). The great unraveling: Losing our way in the new century. WW Norton \& Company. En: http://www.krugmanonline.com/books/the-great-unraveling-losing-our-way-in-the-new-century.php

Langenstein, L., Užík, M., \& Warias, R. (2021). Winner Strategies in Crisis. In SHS Web of Conferences (Vol. 92). EDP Sciences. En: DOI:10.1051/shsconf/20219203015

Lucey, B. M., \& Li, S. (2015). What precious metals act as safe havens, and when? Some US evidence. Applied Economics Letters, 22(1), 35-45. En: https://doi.org/10.1080/13504851.2014.920471

Malkiel, B. G., \& Fama, E. F. (1970). Efficient capital markets: A review of theory and empirical work. The journal of $\quad$ Finance, 25(2), 383-417. En: http://efinance.org.cn/cn/fm/Efficient\%20Capital\%20Markets\%20A\%20Review\%20of\%20Theory\%2 0and\%20Empirical\%20Work.pdf

Narayan, P. K., Phan, D. H. B., \& Liu, G. (2021). COVID-19 lockdowns, stimulus packages, travel bans, and stock returns. Finance research letters, 38, 101732. En: https://doi.org/10.1016/j.frl.2020.101732

O'Brien, P. C., \& Tian, Y. (2006). Financial Analysts' Role in the 1996-2000 Internet Bubble. Available at SSRN 964311. En: https://dx.doi.org/10.2139/ssrn.964311

Organisation for Economic Co-operation and Development (OECD) (2020) Coronavirus (COVID-19): SME policy responses. En: http://www.oecd.org/coronavirus/policy-responses/coronavirus-covid19-smepolicy-responses-04440101/

Öztek, M. F., \& Öcal, N. (2017). Financial crises and the nature of correlation between commodity and stock

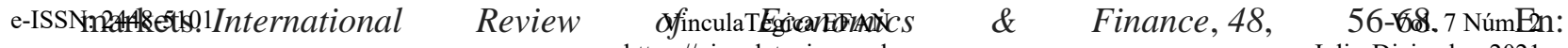


https://www.sciencedirect.com/science/article/abs/pii/S1059056016302830

Pástor, L., \& Veronesi, P. (2006). Was there a Nasdaq bubble in the late 1990s?. Journal of Financial Economics, 81(1), 61-100. En: https://doi.org/10.1016/j.jfineco.2005.05.009

Pena-Marin, J., Adaval, R., \& Shen, L. (2020). Fear in the Stock Market: How COVID-19 Affects Preference for High-and Low-Priced Stocks. https://www.journals.uchicago.edu/doi/abs/10.1086/711930?journalCode=jacr

Penman, S. H. (2002). The quality of financial statements: Perspectives from the recent stock market bubble. En: https://dx.doi.org/10.2139/ssrn.319262

Rosser, J. B. (2000). From Catastrophe to Chaos: A General Theory of Economic Discontinuities: Mathematics, Microeconomics and Finance (Vol. 1). Springer Science \& Business Media.

Rachev, S., Jašić, T., Stoyanov, S., \& Fabozzi, F. J. (2007). Momentum strategies based on reward-risk stock selection criteria. Journal of Banking \& Finance, 31(8), 2325-2346. En: https://doi.org/10.1016/j.jbankfin.2007.02.006

Salas, C., Quintana, L., Mendoza, M. Á., \& Valdivia, M. (2020). Distribución del ingreso laboral y la pobreza en México durante la pandemia de la Covid-19. Escenarios e impactos potenciales. El Trimestre Económico, 87(348), 929-962. En: https://doi.org/10.20430/ete.v87i348.1148

Shiller, R. J. (2000). Measuring bubble expectations and investor confidence. The Journal of Psychology and Financial Markets, 1(1), 49-60. En: https://doi.org/10.1207/S15327760JPFM0101_05

Siegel, J. J. (2003). What is an asset price bubble? An operational definition. European financial management, 9(1), 11-24. En: https://doi.org/10.1111/1468-036X.00206

Sneader, K., \& Sternfels, B. (2020). From surviving to thriving: Reimagining the post-COVID-19 return. McKinsey Global Institute. En: https://www.mckinsey.com/ /media/McKinsey/Industries/Public\%20and\%20Social\%20Sector/Our\%2 0Insights/Future\%20of\%20Organizations/From\%20surviving\%20to\%20thriving\%20Reimagining\%20 the $\% 20$ post $\% 20 \mathrm{COVID} \% 2019 \% 20$ return/From-surviving-to-thriving-Reimagining-the-post-COVID19-return.pdf

Sousa, M., \& Pinho, M. (2014). Is Internet industry facing an IPO bubble 2.0? En: https://dx.doi.org/10.2139/ssrn.2533383

Weerakoon, S. M., Jetelina, K. K., \& Knell, G. (2020). Longer time spent at home during COVID-19 pandemic is associated with binge drinking among US adults. The American Journal of Drug and Alcohol Abuse. En: https://doi.org/10.1080/00952990.2020.1832508

WHO - OMS Coronavirus disease 2019 situation report-67. World Health Organ. 2020 En: https://apps.who.int/iris/handle/10665/331613

Zhang, J., Lai, Y., \& Lin, J. (2017). The day-of-the-week effects of stock markets in different countries. Finance Research Letters, 20, 47-62. En: https://www.sciencedirect.com/science/article/abs/pii/S1544612316301611

Zhang, D., \& Broadstock, D. C. (2018). Global financial crisis and rising connectedness in the international commodity markets. International Review of Financial Analysis, 101239. En: https://www.sciencedirect.com/science/article/abs/pii/S1057521918304587 